versus open lobectomy for clinical stage I lung cancer. Ann Thorac Surg 2013; 96:943-49.

5. van den Berg LL et al. Patterns of Recurrence and Survival after Surgery or Stereotactic Radiotherapy for Early Stage NSCLC. J Thorac Oncol 2015; 10:826-31.

6. Zheng $X$ et al. Survival outcome after stereotactic body radiation therapy and surgery for stage I non-small cell lung cancer: a meta-analysis. Int J Radiat Oncol Biol Phys 2014; 90:603-611.

7. Haasbeek CJ et al. Early-stage lung cancer in elderly patients: a population-based study of changes in treatment patterns and survival in the Netherlands. Ann Oncol 2012; 23:2743-47.

8. National Comprehensive Cancer Network ${ }^{\oplus}$ NCCN Clinical Practice Gui- delines in Oncology (NCCN Guidelines ${ }^{\circledR}$ ) Non-Small Cell Lung Cancer. Version 2.2017 - October 26, 2016. Download am 02. November 2016 von https://www.nccn.org/professionals/physician_gls/pdf/nscl.pdf

9. Vansteenkiste J et al. Early and locally advanced non-small-cell lung cancer (NSCLC): ESMO Clinical Practice Guidelines for diagnosis, treatment and follow-up. Ann Oncol 2013; 24 Suppl 6:vi89-98.

Quelle: Symposium "Heiße Eisen in der Thoraxchirugie 2016" am 30.09.2016 im Rahmen der 25. Jahrestagung der Deutschen Gesellschaft für Thoraxchirurgie und 4. D-A-CH-Tagung, Freiburg.

\title{
Mehr Komplettremissionen dank CAD und Laser
}

\begin{abstract}
Computerassistierte Detektion und lasergestützte Resektion gehören heute zum Standard in der Behandlung von Patienten mit Lungenmetastasen. Sie scheinen einen wichtigen Beitrag zu leisten, um bei möglichst vielen Patienten eine komplette Metastasenresektion zu erzielen.
\end{abstract}

Können Lungenmetastasen komplett reseziert werden, dann verbessert das im Vergleich zur inkompletten Resektion signifikant die Überlebensprognose des Patienten. Das ist seit fast 20 Jahren anhand umfangreicher, internationaler Registerdaten belegt ( $\wedge$ Abb. 1). [1] Verbesserte Detektions- und Resektionstechniken tragen dazu bei, dass bei immer mehr Betroffenen eine komplette Resektion erreicht werden kann.

\section{CAD: Jeder sechste Herd ist nicht palpabel}

Die Computerassistierte Detektion (CAD) ist ein Verfahren zur vertieften Analyse von Bildgebungsdaten, das unter anderem bei der Diagnostik von Lungenmetastasen wertvolle Informationen, zusätzlich zur visuellen Bildauswertung, liefern kann. Dr. med. Barbara Danzl-Erne, Evangelische Lungenklinik Berlin, berichtete aus einer Fallserie von 331 Patienten, bei denen 481 pulmonale Metastasenresektionen nach vorheriger CADAnalyse von Dünnschicht-CTs durchgeführt worden waren. $17 \%$ der damit detektierten Herde konnten intraoperativ nicht palpiert werden. Bei $43 \%$ der Patienten wurden mehr Herde palpiert als via CAD detektiert. „Die CAD ergänzt den Tastsinn, ersetzt ihn aber nicht“, resümierte Danzl-Erne.

\section{Laser: Auch größere Metastasenzahlen behandelbar} Dr. med. Thomas Osei-Agyemang, Klinik für Thoraxchirurgie, Universitätsklinikum Freiburg, stellte eine Fallserie von $223 \mathrm{~Pa}$ tienten vor, bei denen pulmonale Metastasen eines kolorektalen Karzinoms reseziert wurden. Bei 81 Patienten wurde dazu ein Laser eingesetzt. Diese Subgruppe wies durchschnittlich 5,7 Metastasen auf, gegenüber 2,05 Metastasen bei den ohne Laser Operierten $(\mathrm{p}<0,001)$. „Die Metastasenanzahl beeinflusst die OP-Technik“, schloss Osei-Agyemang. Wie zu erwarten, korrelierte die Überlebensdauer mit der Metastasenzahl. Hinsichtlich des Überlebens zeigte sich jedoch kein signifikanter Unter- schied zwischen den mit und ohne Laser Operierten: Die Fünfjahres-Überlebensrate betrug $55 \%$ bei den Resektionen ohne Laser und $47 \%$ bei denen mit Laser ( $\mathrm{p}=0,359)$. Osei-Agyemangs Fazit: „Wenn eine vollständige Metastasenresektion mit Laser technisch machbar ist, dann ist auch bei multiplen Metastasen ein Langzeitüberleben möglich.“

Dr. med. Thomas M. Heim

Literatur:

1. Pastorino $U$ et al. Long-term results of lung metastasectomy: prognostic analyses based on 5206 cases. J Thorac Cardiovasc Surg 1997; 113:37-49.

Quelle: Symposium „Lungenmetastasen und septische Thoraxchirurgie“ am 29.09.2016, im Rahmen der 25. Jahrestagung der Deutschen Gesellschaft für Thoraxchirurgie und 4. D-A-CH-Tagung, Freiburg.

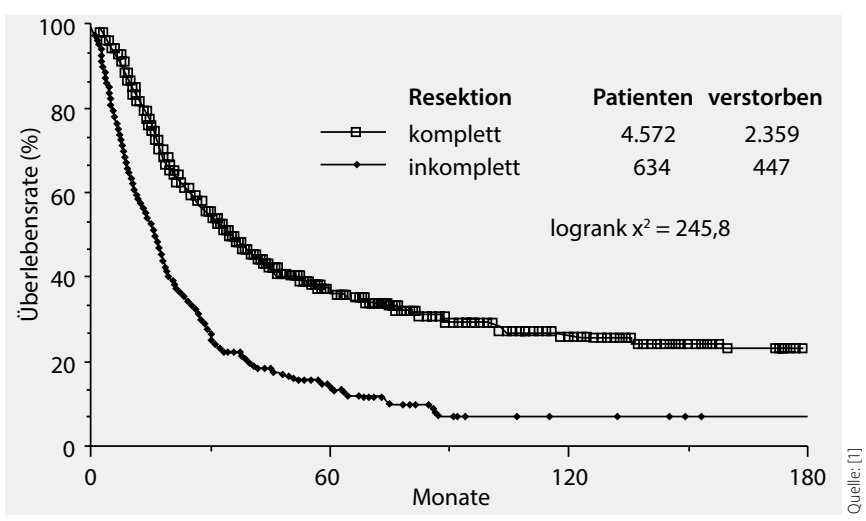

1 Komplette Resektion von Lungenmetastasen verbessert die Überlebensprognose 